\title{
Li and Be depletion in metal-poor subgiants ${ }^{\star}$
}

\author{
A. E. García Pérez ${ }^{1, \star \star}$ and F. Primas ${ }^{2}$ \\ 1 Department of Astronomy and Space Physics, Uppsala University, Box 515, 75120 Uppsala, Sweden \\ e-mail: aegp@astro.uu.se; A.E.Garcia-Perez@open.ac.uk \\ 2 European Southern Observatory, Karl-Schwarzschild Strasse 2, 85748 Garching bei München, Germany \\ e-mail: fprimas@eso.org
}

Received 3 April 2005 / Accepted 7 October 2005

\section{ABSTRACT}

A sample of metal-poor subgiants has been observed with the UVES spectrograph at the Very Large Telescope and abundances of Li and Be have been determined. Typical signal-to-noise per spectral bin values for the co-added spectra are of the order of $500 \mathrm{for}$ the LiI line $(670.78 \mathrm{~nm})$ and 100 for the Be II doublet lines $(313.04 \mathrm{~nm})$. The spectral analysis of the observations was carried out using the Uppsala suite of codes and MARCS (1D-LTE) model atmospheres with stellar parameters from photometry, parallaxes, isochrones and Fe II lines. Abundance estimates of the light elements were corrected for departures from local thermodynamic equilibrium in the line formation. Effective temperatures and $\mathrm{Li}$ abundances seem to be correlated and $\mathrm{Be}$ abundances correlate with $[\mathrm{O} / \mathrm{H}]$. Standard models predict $\mathrm{Li}$ and $\mathrm{Be}$ abundances approximately one order of magnitude lower than main-sequence values which is in general agreement with the observations. On average, our observed depletions seem to be 0.1 dex smaller and between 0.2 and 0.4 dex larger (depending on which reference is taken) than those predicted for $\mathrm{Li}$ and $\mathrm{Be}$, respectively. This is not surprising since the initial $\mathrm{Li}$ abundance, as derived from main-sequence stars on the Spite plateau, may be systematically in error by 0.1 dex or more, and uncertainties in the spectrum normalisation and continuum drawing may affect our Be abundances systematically.

Key words. stars: abundances - stars: atmospheres - stars: late type - stars: Population II

\section{Introduction}

Stellar abundances of the very light elements lithium, beryllium, and boron ( $\mathrm{Li}, \mathrm{Be}$, and $\mathrm{B})$ have been studied extensively during the recent decades, mainly because of the great role they play in constraining Big Bang nucleosynthesis theories as well as models of galactic chemical evolution and stellar evolution.

Stellar evolution predicts depletion of the light elements in stellar atmospheres under certain physical conditions. Stars with their surface convection base reaching depths where there has been some depletion should reflect such depletion in their stellar surfaces. The convective motions can bring up material from the interior to the surface, i.e. gas poor in light elements is mixed with gas with the initial composition, leading to dilution, this is expected to happen in subgiant stars which are the type of stars under study in this work. If the temperature at the base of the convection zone is high enough, light elements brought down from the surface can be destroyed.

With this background in mind, we have looked at lithium and beryllium abundances in a sample of metal-poor subgiant

\footnotetext{
^ Based on observations collected at the European Southern Observatory, Chile (ESO No. 68.D-0546).

$\star \star$ Student visitor at the European Southern Observatory, Munich, Germany.
}

stars, from a new set of UVES observations. The analysis of the Be features, in crowded spectral regions where blending may be a serious problem, requires detailed spectrum synthesis of high quality.

Standard models, e.g. Deliyannis et al. (1990), include only convection as the mixing mechanism. According to these models, depletion occurs at low stellar mass and low effective temperatures. These stars have convection zones deep enough to reach inner layers where the light elements have been depleted. The models predict burning of the light isotopes during the premain sequence (PMS) phase and dilution in the subgiant evolutionary phase. The fact that the Sun has a photospheric logarithmic $\mathrm{Li}$ abundance of $A(\mathrm{Li})=1.05$ (Asplund et al. 2005) which is significantly lower than the values measured in meteoritic matter (which is thought to be representative of the gas at the time our solar system formed) indicates that also mainsequence (MS) stars undergo some mixing process (other than simple convection).

On the main-sequence, diffusion (Michaud 1986; Richard et al. 2002), rotation induced mixing (Pinsonneault et al. 1990), mass loss (Schramm et al. 1990; Vauclair \& Charbonnel 1995) and gravity waves (García López \& Spruit 1991; Talon \& Charbonnel 2004) have been proposed to explain the light element patterns observed in MS stars. Light elements burn at low, 
but different, burning temperatures. The most fragile of them is $\mathrm{Li}$ (with a burning temperature $T_{\mathrm{b}} \simeq 2.5 \times 10^{6} \mathrm{~K}$ ), followed by $\mathrm{Be}\left(T_{\mathrm{b}} \simeq 3.0 \times 10^{6} \mathrm{~K}\right)$ and $\mathrm{B}\left(T_{\mathrm{b}} \simeq 5.0 \times 10^{6} \mathrm{~K}\right)$. Therefore, if at least the abundances of two of the light isotopes are known in a star, their abundances may be used to further constrain which type of internal mixing is active in the stellar external layers. Models with only mass loss fail to predict a simultaneous Li and Be depletion, i.e. lithium is expected to be completely depleted before beryllium shows any sign of depletion. However observations show just the opposite, i.e. some degree of Be depletion in stars in which Li can still be detected. One such example (there are several other cases) are the Hyades F stars, which show depletion in both Li and Be (Boesgaard \& Tripicco 1986; Deliyannis et al. 1998).

As for the case of main-sequence stars, the knowledge of two or more light isotopes in subgiants can help us to better understand the mixing processes occurring in the atmospheres of these stars. In this work, we have exploited that possibility by determining both $\mathrm{Li}$ and $\mathrm{Be}$ abundances and comparing the observed abundance depletions with predictions based on standard models.

This comparison requires the assumption of a value for the initial abundances so that the magnitude of the observed depletion can be quantified. About $70 \%$ of the ${ }^{7} \mathrm{Li}$ observed in the early Galaxy is synthesised during the Big Bang (Walker et al. 1991, 1993; Olive et al. 2000). Some extra contributions by $\alpha-\alpha$ reactions during late stellar evolutionary stages, like (post-)AGB stars and novae, contribute at later epochs to the enrichment of the Galaxy. These sources influence the galactic evolution of lithium at higher metallicities. Therefore, ${ }^{7} \mathrm{Li}$ abundances in the oldest, most metal-poor stars of the Galactic halo are of great importance from a cosmological point of view, providing useful constraints on the cosmological baryon-to-photon ratio $\eta$. In the early 1980 's, Spite \& Spite (1982) found a constant logarithmic lithium abundance, $A(\mathrm{Li})=2.1^{1}$, in a small sample of warm metal-poor mainsequence stars spanning a wide range of (low) metallicities, the so called Spite lithium plateau. Although the plateau has now been confirmed by large data samples, the interpretation of its constant value (whether $\mathrm{Li}$ is primordial or not) still remains an open question, as well as if it is tilted with respect to metallicity and effective temperature (cf. Charbonnel \& Primas 2005, for a recent review). If this plateau value represents the primordial abundance of $\mathrm{Li}$, then one needs to explain why in the Galaxy today its abundance is 3.25 (Asplund et al. 2005).

For the other light elements, Reeves et al. (1970) and Meneguzzi et al. (1971) proposed cosmic-ray spallation reactions as the main (if not unique, as in the case of beryllium) source of ${ }^{6} \mathrm{Li},{ }^{9} \mathrm{Be}$ and ${ }^{10,11} \mathrm{~B}$ production. In this scenario, light elements are produced via collisions of $\mathrm{C}$ and $\mathrm{O}$ target nuclei with protons and $\alpha$ particles in the interstellar medium. This predicts a quadratic dependence of the light isotope abundance on the target nuclei, e.g. oxygen. However, in the early 1990's, Gilmore et al. (1992) (for Be) and Duncan et al. (1992) (for B) found a linear slope, which has triggered the exploration of several variations of the standard cosmic-ray spallation

\footnotetext{
1 Abundances are denoted by $A(\mathrm{X})=\log \left(N_{\mathrm{X}} / N_{\mathrm{H}}\right)+12$.
}

theory. The observed linear trend (further confirmed by larger data samples, Molaro et al. 1997; Boesgaard et al. 1999) seems to imply a primary origin of Be and $\mathrm{B}$ instead of a secondary one. One possibility to achieve this is if $\mathrm{C}$ and $\mathrm{O}$ nuclei were freshly synthesised by type II supernovae (SNeII) and accelerated by the outwards shocks together with the $\alpha$ particles and protons, and different scenarios more or less built on this idea have been exploited (Duncan et al. 1992; Feltzing \& Gustafsson 1994; Cassé et al. 1995; Parizot \& Drury 1999). Knowledge of the correlation between $\mathrm{Be}$ and $\mathrm{O}$ stellar abundances in larger samples of stars will help us to discern between these different scenarios (it should be noted that many more models have been developed in the last few years, and that those mentioned above represent some main ideas, which have subsequently been developed with different degrees of sophistication).

On the observational side, in general, the abundances of light elements in stars are so small that the only lines used for abundance determination purposes are resonance lines. The observations of these lines as well as their modelling have been quite a challenge. Most studies have so far concentrated on lithium; its resonant doublet at $670.78 \mathrm{~nm}$ makes it easily accessible with ground based telescopes. Beryllium, instead, having its main resonant doublet at $313.0 \mathrm{~nm}$, requires high resolution and highly efficient spectrographs in the near-UV. Boron, the third light element, is even more challenging as it may be studied only from space (such as with the Hubble Space Telescope), as its main resonant doublet falls at $250.0 \mathrm{~nm}$. Thanks to the very efficient high-resolution spectrographs currently available, we note that observations of ${ }^{6} \mathrm{Li}$ in Galactic stars have become more accurate and affordable in terms of telescope time (Nissen et al. 2000; Aoki et al. 2004, and references therein).

In the following sections we describe the observations as well as the stellar parameters and stellar abundances determinations, which are then discussed in the last section in terms of dilution in subgiants.

\section{Observations and data reduction}

Our sample of metal-poor subgiants was observed in service mode between October 2000 and March 2001, with the Ultraviolet Visual Echelle Spectrograph (UVES, Dekker et al. 2000), mounted on the Nasmyth B platform of the $8 \mathrm{~m}$ Kueyen unit of the ESO Very Large Telescope (VLT). All observations were taken in dichroic mode, i.e. using both the red and the blue arms of the spectrograph simultaneously, thus covering a wide range in wavelength. The instrument set-ups chosen were the Dichroic\#1 (Blue346 combined with Red580, where 346 and 580 denote the central wavelengths in $\mathrm{nm}$ of the two settings), and Dichroic \#2 (Blue346 plus Red860). In this way we obtained a spectral coverage from 300 to $380 \mathrm{~nm}$ in the blue, and from 480 to $1000 \mathrm{~nm}$ in the red (with the only exception of the narrow gaps between the two CCDs used in the UVES Red Arm).

The observations had been proposed and planned for a detailed study of oxygen abundances using three different abundance indicators ([OI], OI, and the $\mathrm{OH}$ lines in the near-UV, García Pérez et al. 2005), but the large spectral coverage also 
Table 1. Stellar parameters of our programme stars as derived by García Pérez et al. (2005).

\begin{tabular}{lcccc}
\hline \hline Star & $\begin{array}{c}T_{\text {eff }} \\
{[\mathrm{K}]}\end{array}$ & $\begin{array}{c}\log g \\
{[\mathrm{cgs}]}\end{array}$ & $\begin{array}{c}{[\mathrm{Fe} / \mathrm{H}]} \\
{[\mathrm{dex}]}\end{array}$ & $\begin{array}{c}\xi_{\text {micro }} \\
{\left[\mathrm{km} \mathrm{s}^{-1}\right]}\end{array}$ \\
\hline HD 4306 & 4990 & $3.04 \pm 0.26$ & -2.33 & 1.5 \\
HD 26169 & 4972 & $2.49 \pm 0.26$ & -2.28 & 1.5 \\
HD 27928 & 5044 & $2.67 \pm 0.43$ & -2.14 & 1.5 \\
HD 45282 & 5352 & $3.15 \pm 0.13$ & -1.52 & 1.1 \\
HD 108317 & 5300 & $2.76 \pm 0.22$ & -2.25 & 1.5 \\
HD 126587 & 4712 & $1.66 \pm 0.50$ & -2.87 & 1.5 \\
HD 128279 & 5336 & $2.95 \pm 0.21$ & -2.19 & 1.5 \\
HD 200654 & 5292 & $2.86 \pm 0.35$ & -2.73 & 1.5 \\
HD 218857 & 5015 & $2.78 \pm 0.36$ & -1.79 & 1.4 \\
HD 274939 & 5090 & $2.79 \pm 0.33$ & -1.49 & 1.3 \\
BD - 01 2582 & 5072 & $2.92 \pm 0.40$ & -2.11 & 1.5 \\
CD - 24 1782 & 5228 & $3.46 \pm 0.35$ & -2.25 & 1.5 \\
CD - 30 0298 & 5196 & $2.93 \pm 0.50$ & -3.01 & 1.5 \\
\hline
\end{tabular}

allowed us to study other elements like the light elements, lithium and beryllium. The need for detecting the three different oxygen indicators in the same object restricted this study to a relatively narrow range of surface gravities and effective temperatures (see Table 1), which corresponds to stars that have already left the MS and started evolving (ascending) along the subgiant (giant) branch. Moreover, accurate oxygen abundances determinations require accurate surface gravity determinations. Hence, our stars were chosen based on the availability of Hipparcos parallaxes from which surface gravities were estimated. The selected stars ended up being not very distant objects (the parallaxes of most of them indicate distances smaller than $400 \mathrm{kpc}$ ) which reduces the effective temperature uncertainties due to reddening.

Lithium is easily measurable from its resonant doublet at $670.78 \mathrm{~nm}$, whereas beryllium is more challenging as its main resonant doublet falls at $313.0 \mathrm{~nm}$, i.e. in a very crowded region and very close to the atmospheric cut-off. In order to maximise the signal-to-noise ratio of the near-UV spectra, a $2 \times 2$ binning was selected when reading out the EEV chip of the UVES blue arm. This choice did not affect the resolution of our final spectra since we chose a slit width of $1^{\prime \prime}$, the nominal resolving power of which $(R=45000)$ implies some oversampling. In the red arm, we selected a slit width of $0.7^{\prime \prime}$ corresponding to a nominal resolving power of $R=55000$, thus resolving the blending of the Li line by an Fe I line at $670.74 \mathrm{~nm}$ (in spectra for which such blending could be a problem, i.e. for the more metal-rich stars). Typical signal-to-noise $(S / N)$ per spectral bin values are of the order of 100 and 500 at the $\mathrm{Be}$ and $\mathrm{Li}$ lines, respectively, in the final co-added spectra.

Data reduction followed standard reduction procedures, i.e. bias subtraction, division by a normalised flat-field, extraction of the echelle orders and their wavelength calibration. After a careful inspection of the blue spectra, we decided that the quality achieved by the UVES data reduction pipeline processing was satisfactory for our purposes. However, for the red spectra, we decided to manually re-reduce all the spectra (using IRAF) in order to improve (i.e. minimise) the presence of fringes and ripples in these very high $S / N$ spectra. We then normalised all
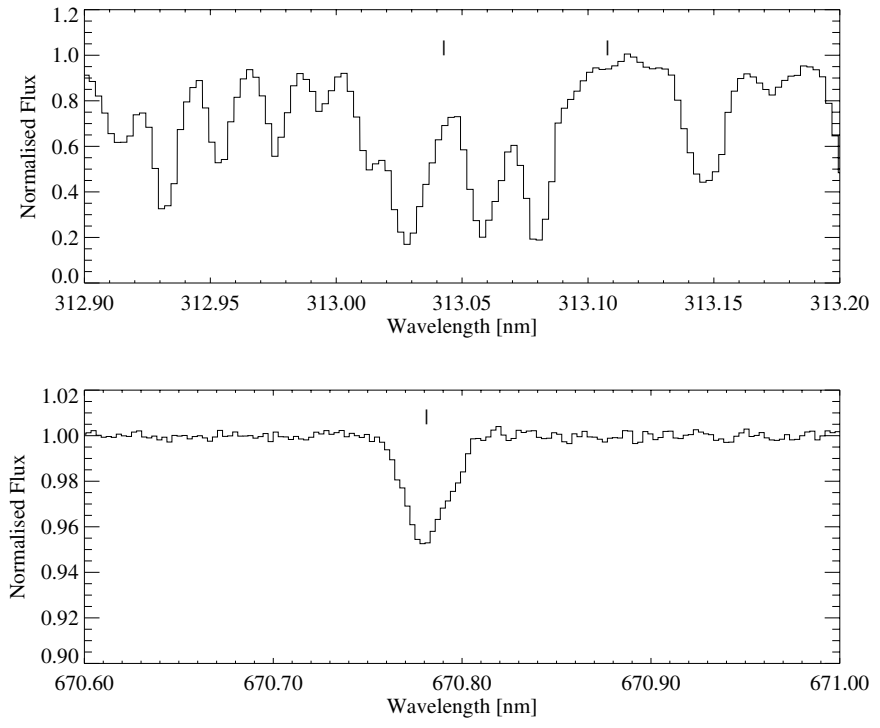

Fig. 1. Spectra of the Be (top) and Li (bottom) regions in one star of our sample, HD 218857. The positions of the Be II doublet and of the Li I line are indicated by vertical bars in both panels.

the spectra with a spline function. This step is straightforward in the lithium region (where the stellar continuum is easily identifiable), but the severe spectral-line crowding in the nearUV region does not allow such an easy identification. Instead, the near-UV spectra were normalised by using synthetic spectra that were computed for each individual star.

In spite of the weakness of the observed lines, the very high quality of our UVES spectra allowed us to detect both the $\mathrm{Li}$ and the Be lines in almost all stars. Figure 1 illustrates the spectral quality of one of our targets, HD $218857(V=8.967)$.

\section{Stellar parameters and model atmospheres}

\subsection{Stellar parameters}

Since the determination of the stellar parameters for our sample of stars is presented in a parallel paper (García Pérez et al. 2005), here we will only summarise how they were derived. Table 1 lists the values used for the effective temperature $\left(T_{\text {eff }}\right)$, the logarithmic surface gravity $(\log g)$, the metallicity $([\mathrm{Fe} / \mathrm{H}])$ and the microturbulence.

The process of determining stellar parameters is clearly an iterative process. In short, $T_{\text {eff }}$ was estimated from the $(b-y)$ and $(V-K)-T_{\text {eff }}$ calibrations of Alonso et al. (1996), based on the Infrared Flux Method (IRFM). Strömgren (uvby $\beta)$ and $V$, $K$ photometry were taken from Schuster \& Nissen 1988, and private communication) and the Two Micron All Sky Survey (2MASS) database, respectively. Colours and magnitudes were de-reddened with values estimated from the Hakkila et al. (1997) galactic maps of interstellar reddening.

The surface gravity was derived from Hipparcos parallaxes following the prescription in Nissen et al. (1997). Parallaxes are available for all programme stars and, furthermore, their uncertainties are all very small, except for two stars, HD 126587 and $\mathrm{CD}-30^{\circ} 0298$, for which the uncertainty is as large or even larger than the parallax itself. Therefore, the surface gravities 
Table 2. Lithium abundances of the programme stars. Equivalent widths $\left(W_{\lambda}\right)$ of the Li feature at $670.78 \mathrm{~nm}$ are given in the second column, followed in the third column by the lithium abundances. Columns 4 to 7 show the sensitivity of the lithium abundances to changes in the stellar parameters and in the equivalent width (varied by their typical uncertainties). Final errors based on the square sum of these values are listed in Col. $8(\sigma)$. NLTE Li abundances as derived from the MULTI code of Carlsson et al. (1994) and the oxygen abundances as derived from the O I lines at $630.03 \mathrm{~nm}$ (cf. García Pérez et al. 2005) are given in the two last columns.

\begin{tabular}{lccccccccc}
\hline \hline Star & $\begin{array}{c}W_{\lambda} \\
{[\mathrm{pm}]}\end{array}$ & $\begin{array}{c}A(\mathrm{Li}) \\
\mathrm{LTE}\end{array}$ & $\begin{array}{c}\Delta A(\mathrm{Li}) \\
\left(T_{\text {eff }}\right)\end{array}$ & $\begin{array}{c}\Delta A(\mathrm{Li}) \\
(\log g)\end{array}$ & $\begin{array}{c}\Delta A(\mathrm{Li}) \\
([\mathrm{Fe} / \mathrm{H}])\end{array}$ & $\begin{array}{c}\Delta A(\mathrm{Li}) \\
\left(W_{\lambda}\right)\end{array}$ & $\sigma$ & $\begin{array}{c}A(\mathrm{Li}) \\
\text { NLTE }\end{array}$ & $\begin{array}{c}A(\mathrm{O}) \\
\text { LTE }\end{array}$ \\
\hline HD 4306 & 1.63 & 0.97 & 0.11 & 0.00 & 0.00 & 0.02 & 0.11 & 1.06 & 7.14 \\
HD 26169 & 1.44 & 0.91 & 0.11 & -0.01 & 0.00 & 0.02 & 0.11 & 1.08 & 7.05 \\
HD 27928 & 1.36 & 0.96 & 0.10 & -0.02 & -0.01 & 0.01 & 0.10 & 1.12 & 7.06 \\
HD 45282 & 1.03 & 1.14 & 0.10 & 0.00 & 0.00 & 0.02 & 0.10 & 1.24 & 7.72 \\
HD 108317 & $<0.08$ & $<0.00$ & 0.09 & -0.01 & 0.00 & $<0.18$ & 0.20 & $<0.11$ & 7.23 \\
HD 126587 & 1.75 & 0.76 & 0.11 & -0.03 & 0.00 & 0.01 & 0.11 & 0.96 & 6.32 \\
HD 128279 & 1.06 & 1.13 & 0.09 & 0.00 & 0.00 & 0.02 & 0.09 & 1.24 & 6.96 \\
HD 200654 & 1.10 & 1.09 & 0.10 & 0.00 & 0.01 & 0.03 & 0.10 & 1.20 & $<6.75$ \\
HD 218857 & 1.33 & 0.90 & 0.12 & -0.01 & 0.00 & 0.02 & 0.12 & 1.06 & 7.33 \\
HD 274939 & 1.44 & 1.02 & 0.11 & -0.01 & 0.00 & 0.02 & 0.11 & 1.17 & 7.76 \\
BD - 01 2582 & 0.63 & 0.63 & 0.10 & -0.01 & 0.00 & 0.03 & 0.10 & 0.77 & 7.17 \\
CD - 24 1782 & 1.12 & 1.05 & 0.09 & 0.00 & 0.00 & 0.02 & 0.09 & 1.16 & 6.80 \\
CD - 30 0298 & 1.01 & 0.96 & 0.09 & 0.00 & 0.00 & 0.02 & 0.09 & 1.08 & $<6.38$ \\
\hline
\end{tabular}

of these two stars were estimated from a colour-magnitude diagram using VandenBerg et al. (2000) evolutionary tracks. As described in García Pérez et al. (2005), this method was applied to the entire sample, as a consistency check on the $\log g$ values derived from the parallaxes. A mass of $0.8 M_{\odot}$ was assumed for all the stars and the bolometric corrections were taken from Bessell et al. (1998).

The metallicity values reported in Table 1 are spectroscopic values, and they have been determined from the equivalent widths of a set of Fe II lines (for more details see García Pérez et al. 2005). This same set of iron lines was used to constrain the microturbulence, by minimising the dependence of the iron abundance on the equivalent widths of the lines.

\subsection{Model atmospheres}

In order to model the stellar atmospheres of the programme stars, the equation of hydrostatic equilibrium, the equation of radiative transfer and the flux-constancy condition, with mixing-length convective flux, were solved simultaneously for the set of stellar parameters of each of the stars (cf. Table 1), using an updated version of the MARCs code (Asplund et al. 1997). In order to solve the equations (in one dimension and assuming local thermodynamic equilibrium (LTE) conditions), the program requires in addition to the stellar parameters listed above also abundances of certain elements such as $\mathrm{C}, \mathrm{N}, \mathrm{O}$, $\mathrm{Ne}, \mathrm{Na}, \mathrm{Mg}$, etc. These elements need to be taken into account when computing the number of electrons and opacities in stellar atmospheres and their relative abundances were chosen to be solar (Grevesse \& Sauval 1998), scaled to the stellar metallicity; however, the solar oxygen abundance was taken from the 1D-LTE analysis of the oxygen forbidden line at $630.03 \mathrm{~nm}$ (Nissen et al. 2002), and a value of 0.4 dex was assumed for the $\alpha$-enhancement of Population II stars. No enhancement was applied to the $\mathrm{C}$ abundance.

\section{Abundance analysis and uncertainties}

\subsection{Lithium abundances}

The Li line at $670.78 \mathrm{~nm}$ lies in a spectral region which is almost free of other lines, making it possible in all our spectra to easily identify a clean wavelength region of $\sim 0.4 \mathrm{~nm}$ around the Li line and to unambiguously define the stellar continuum. This, together with the fact that the blending by a neighbouring Fe I line at $670.74 \mathrm{~nm}$ is insignificant at our low metallicities, makes the measurement of the equivalent widths very accurate. The widths were measured by integrating the area under the line using the IRAF task splot and assuming a Gaussian profile. Direct integration, without the profile assumption, gave very similar results. The values are given in Col. 2 of Table 2. There is only one star, HD 108317, for which the line could not be detected. A value of $0.08 \mathrm{pm}$ was estimated as an upper limit to its equivalent width based on a two sigma observational error. The associated abundance must therefore be considered an upper limit.

The lithium line at $670.78 \mathrm{~nm}$ is a multiplet but the resolution of our observed red spectra is not high enough to resolve it completely. The feature was treated as a single line when solving the radiative transfer equation. The oscillator strength used in our analysis $(\log g f=0.17)$ was the sum of the values for the individual components given in Table 3 of Smith et al. (1993). 1D-LTE abundances were derived from the measured equivalent widths using the EQW code from the Uppsala package of stellar atmospheres. As a cross-check, we also ran some spectral syntheses and found that in general there is a very good agreement between the lithium abundances derived by the two methods. However, it is well known that the Li I line at $670.78 \mathrm{~nm}$ forms under non-LTE conditions (NLTE). Therefore, NLTE corrections should be applied to the LTE abundances. For the stars analysed here, these corrections were estimated using the routines and the NLTE results in Carlsson et al. (1994). The final NLTE Li abundances (i.e. 
Table 3. Our final Be abundances as determined from the spectral synthesis fitting of the Be doublet (Col. 2). The sensitivity of the result to changes in the stellar parameters (corresponding to their uncertainties) are presented in Cols. 3 to 5 and their associated $\sigma$ values, calculated as in the case of $\mathrm{Li}$, are given in Col. 6. Column 7 lists the final NLTE corrected Be abundances.

\begin{tabular}{lcccccr}
\hline \hline Star & \multicolumn{7}{c}{$A(\mathrm{Be})$} & $\Delta A(\mathrm{Be})$ & $\Delta A(\mathrm{Be})$ & $\Delta A(\mathrm{Be})$ & $\sigma$ & $A(\mathrm{Be})$ \\
& LTE & $\left(T_{\text {eff }}\right)$ & $(\log g)([\mathrm{Fe} / \mathrm{H}])$ & NLTE \\
\hline HD 4306 & -1.84 & -0.02 & 0.17 & 0.03 & 0.17 & -1.84 \\
HD 26169 & -1.65 & 0.00 & 0.15 & 0.02 & 0.15 & -1.65 \\
HD 27928 & -1.96 & -0.01 & 0.26 & 0.02 & 0.26 & -2.00 \\
HD 45282 & -1.15 & 0.00 & 0.07 & 0.03 & $0.08-1.18$ \\
HD 108317 & $<-2.30$ & 0.03 & 0.10 & 0.01 & $0.10<-2.26$ \\
HD 126587 & -2.58 & 0.02 & 0.30 & 0.01 & $0.30-2.37$ \\
HD 128279 & $<-2.01$ & 0.03 & 0.11 & 0.01 & $0.11<-1.94$ \\
HD 200654 & $<-2.06$ & 0.03 & 0.17 & 0.01 & $0.17<-2.04$ \\
HD 218857 & -1.42 & -0.03 & 0.22 & 0.03 & $0.22-1.52$ \\
HD 274939 & -1.20 & -0.03 & 0.20 & 0.03 & $0.20-1.28$ \\
BD - 01 & 2582 & -1.74 & -0.01 & 0.25 & 0.03 & $0.25-1.81$ \\
CD - 24 1782 & -1.45 & 0.00 & 0.21 & 0.02 & $0.21-1.54$ \\
CD - 30 0298 & -2.04 & 0.03 & 0.27 & 0.00 & $0.27-2.04$ \\
\hline
\end{tabular}

LTE Li plus the correction) are listed in Col. 9 of Table 2. The largest NLTE correction is of the order of 0.20 dex. The lithium abundance of HD 108317 and the effective temperature of the giant HD 126587 lie outside the range of $T_{\text {eff }}$ and $A(\mathrm{Li})$ covered in Carlsson et al. (1994). For these two stars, NLTE corrections based on their closest points in the grid of Carlsson et al. have been adopted.

The final uncertainty in each lithium abundance was calculated by taking into account the uncertainty in the equivalent width measurement and in the stellar parameters. According to photon statistics, the associated error in equivalent widths for a $S / N \sim 500$ spectrum is of the order of 0.03-0.04 pm. Here, in order to take into account also the uncertainty due to the continuum placement (although very small) we assumed a total uncertainty of $0.05 \mathrm{pm}$ in each equivalent width. On the average, this corresponds to abundance errors of the order of 0.02 dex only.

The sensitivity to the stellar parameters was computed by testing the response of the derived lithium abundance to changes in $T_{\text {eff }}( \pm 100 \mathrm{~K})$, in $\log g$ ( \pm its $1 \sigma$ errors which are listed in Col. 3 of Table 1$)$, and in $[\mathrm{Fe} / \mathrm{H}]( \pm 0.1 \mathrm{dex})$. The influence of these changes on the final $\mathrm{Li}$ abundance are listed in Cols. 4 to 7 of Table 2. Obviously, the lithium abundances are mostly sensitive to the effective temperature (a 0.1 dex effect). The errors in lithium abundances due to errors in other stellar parameters are negligible.

\subsection{Beryllium abundances}

The beryllium abundances are based on synthetic-spectrum analysis of the Be II resonant doublet at 313.0-1 nm. The bluer component $(\lambda 313.04 \mathrm{~nm})$ is the stronger of the two lines, but it is severely blended (mainly by $\mathrm{Cr}, \mathrm{V}, \mathrm{CH}$ and $\mathrm{OH}$ lines). The other component of the doublet $(\lambda 313.11 \mathrm{~nm})$ is very weak in our programme stars; it lies in the wing of a much stronger $\mathrm{Ti}$ line but it is otherwise almost free of blends. There is no special reason to prefer one line over the other. Instead, spectral synthesis of a region of $0.3 \mathrm{~nm}$ around the two Be lines is mandatory in order to properly account for the blends. The 1D-LTE syntheses were run with the program BSYN of the Uppsala suite of codes. The line list of atomic lines and $\mathrm{CH}$ lines used was the same as the one in Primas et al. (1997), whereas a new OH line list was used, based on Gillis et al. (2001).

The elemental abundances were those adopted for the model atmospheres, except for those elements with transitions in the $0.3 \mathrm{~nm}$ wavelength range that was synthesised. Once computed, each synthetic spectrum was convolved with a profile taking care of macroturbulence, stellar rotation etc., and with a Gaussian with a width corresponding to the resolution of the spectra. The widths of these functions were changed together with the stellar abundances until the best fit was achieved. Our final beryllium abundances (Col. 2, Table 3) correspond to the abundance value that best fits both Be II lines, see Fig. 2.

The formal uncertainty in our Be abundance determinations was derived similarly to what was done in the case of lithium. Here we took only into account the sensitivity of Be to changes (corresponding to $1 \sigma$ uncertainties) in the stellar parameters. We found that the surface gravity determination plays a major role in obtaining accurate Be abundances, leading to uncertainties $A(\mathrm{Be})$ of about 0.2 dex. On the other hand, uncertainties associated to effective temperature and metallicity are insignificant.

An important source of error in the Be abundance is due to the normalisation and continuum location in fitting the synthetic spectra. We estimate that this source of error may well contribute errors in $A(\mathrm{Be})$ of about 0.2 dex. There is also a risk that this error may be systematic, e.g. leading to too small Be abundances for the majority of the stars.

The Be abundances given in Table 3 were determined from the LTE spectral analysis of the Be II UV doublet lines at 313.0$1 \mathrm{~nm}$. However, according to the work of García López et al. (1995) and García Pérez \& Kiselman (2005, in preparation), these lines are formed in the stellar atmospheres under non LTE conditions. Specific NLTE calculations were carried out following the procedure described and discussed by García Pérez and Kiselman (2005, in preparation), using an adapted version of the MULTI code. NLTE Be abundances were determined by matching the equivalent width calculated in LTE with abundances from the spectrum synthesis with the NLTE computed ones. The highest NLTE correction $(0.2 \mathrm{dex})$ is found for the giant star, HD 126587. For this star, the NLTE Li abundance correction is also among the highest.

\section{Results and discussion}

Simultaneous knowledge of lithium and beryllium abundances in the same star is a powerful diagnostic of mixing activity in stellar interiors. According to standard models, halo stars cooler than about $5700 \mathrm{~K}$ on the main-sequence should show Li depletion (which has already taken place during their pre-main-sequence phase). On the contrary, metal-poor stars warmer than this temperature all show a similar Li abundance, the so-called Spite lithium plateau (Spite \& Spite 1982; 


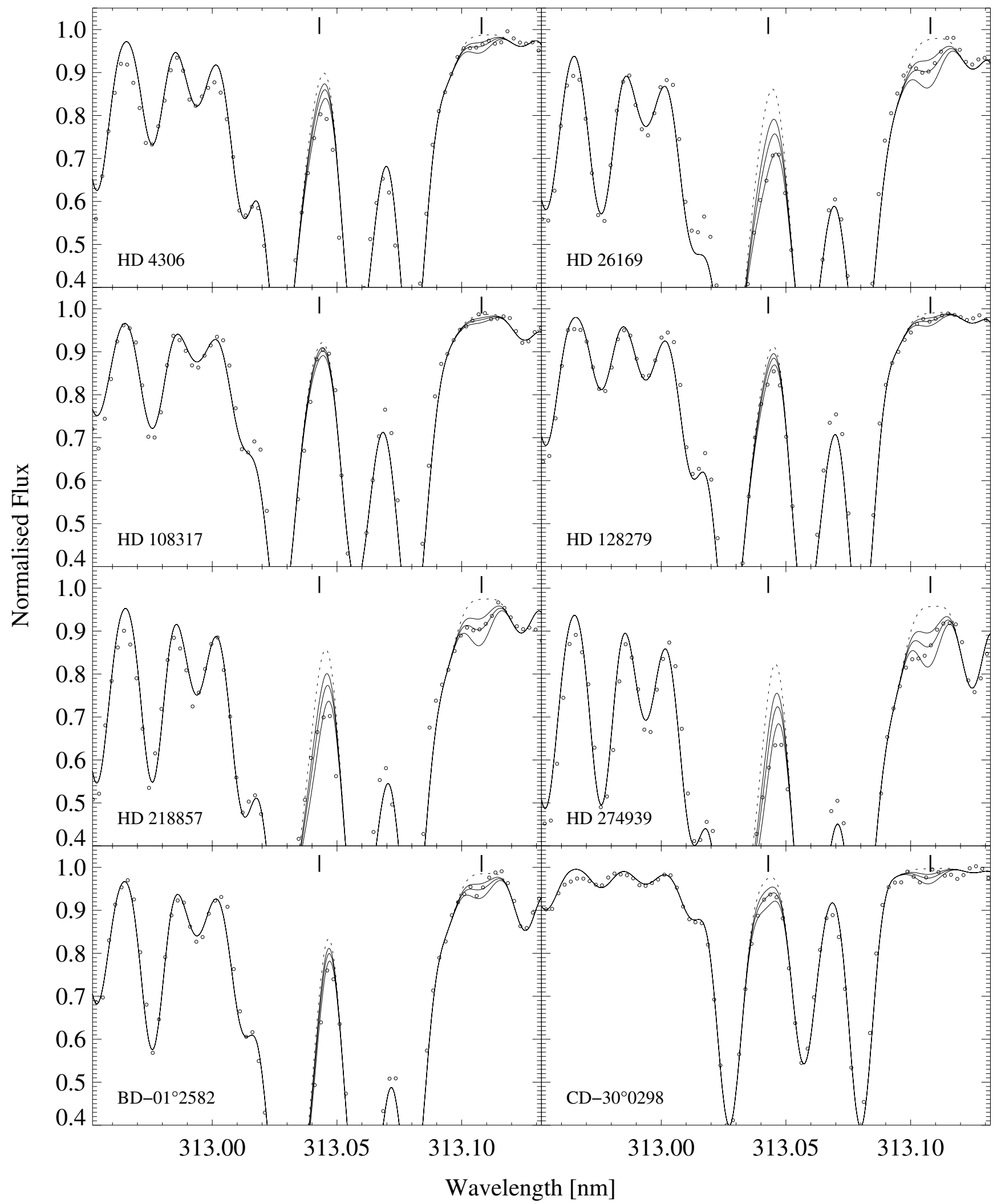

Fig. 2. 1D-LTE spectral syntheses for a selection of 8 programme stars, in the wavelength range around the Be II UV doublet lines at 313.0-1 $\mathrm{nm}$ computed for four different Be abundances: the best-fit abundance, abundance changes by \pm 0.2 dex around that value, and a very low value of the Be abundance (practically zero). The observed spectra are denoted by open circles and the synthetic spectra by solid lines. The dashed line corresponds to the synthetic spectra for the very low value of $A(\mathrm{Be})$. 


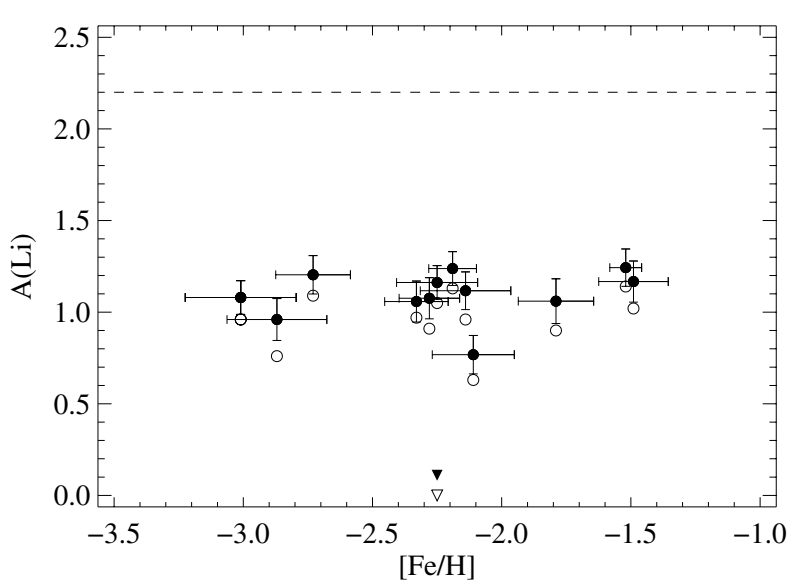

Fig. 3. Logarithmic lithium abundances in a 12-scale as a function of stellar metallicity. Both NLTE (filled circles) and LTE (open circles) abundances are shown. Dashed line shows a 2.2 constant value as representative of metal-poor main-sequence stars in the Spite plateau.

Ryan et al. 1999). According to stellar evolution theory, as stars evolve from dwarfs to giants, they get cooler but the surface convection zone deepens and reaches layers which were hot enough to "burn" $\mathrm{Li}$. The matter poor in $\mathrm{Li}$ is mixed with the rest of the convection zone, so that dilution of lithium is expected.

The surface gravities of our programme stars are typical of subgiants. Their spectra show weak lithium lines which suggest depletion. In one case, HD 108317, the Li line was not even detected. Something similar happens to the Be II UV doublet lines at 313.0-1 nm in subgiants, i.e. they get weaker and more difficult to detect, especially the redder line of the doublet which is the cleaner but unfortunately also the weaker of the two. This line $(\lambda 313.1 \mathrm{~nm})$ was not detected in the observed spectra of HD 108317, HD 128279 and HD 200654. When upper limits are given, they are constrained by synthetic spectrum fits to both lines.

\subsection{Lithium depletion}

As is seen in Figs. 3 and 4, the derived lithium abundances of the programme stars, either the LTE (open circles) or the NLTE (filled circles) values, are well below the Spite plateau value (here taken to be $A(\mathrm{Li})_{\mathrm{NLTE}}=2.20$, close to Bonifacio \& Molaro 1997), by about 1 dex. No obvious correlation is seen between the lithium abundances and metallicity, and the observed difference in $A(\mathrm{Li})$ between stars at the same metallicity can be fully explained by the associated errors, mostly due to uncertainties in the effective temperature.

Assuming that our observed subgiants have left the MS with $\mathrm{Li}$ abundance values typical of the Spite plateau, we can estimate their logarithmic depletion factors using the Spite plateau value as the initial value $\left(A(\mathrm{Li})_{\mathrm{i}}=2.2\right)$. The depletion factors (Col. 2, Table 4) based on that MS value are compared with dilution predictions (Col. 3, Table 4) in Fig. 4 as a function of effective temperature, where the predicted values are based on evolutionary tracks presented in Deliyannis et al. (1990) for a subgiant star with $0.775 M_{\odot}$ and $[\mathrm{Fe} / \mathrm{H}]=-2.3$.

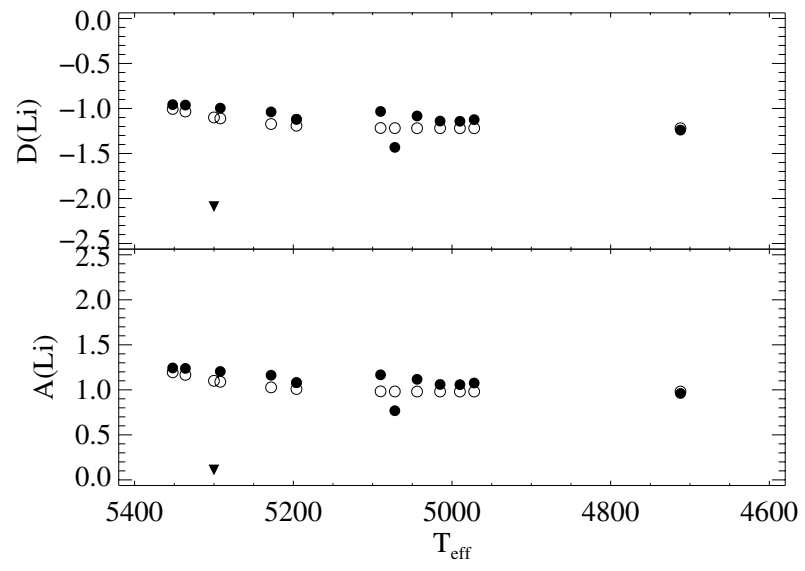

Fig. 4. Logarithmic Li depletion factors (top panel) and Li abundances (bottom panel) as a function of effective temperature. Observed depletion factors and the NLTE Li abundances (filled circles) on which these factors are based are plotted together with the predicted values (open circles). A value of 2.2 was taken as representative of initial $\mathrm{Li}$ abundance. The triangle denotes the star HD 108317 with an upper limit on the $\mathrm{Li}$ abundance.

Table 4. Logarithmic Li and Be depletion factors for the programme stars, based on abundances in metal-poor dwarf stars. For Be, factors associated to two different Be-vs.-O trends are quoted: trend in Primas et al. (2005, in preparation, Col. 4) and in (Boesgaard et al. 1999, Col. 5). Predictions based on Deliyannis et al. (1990) evolutionary tracks are given in Cols. 3 and 6.

\begin{tabular}{lccccc}
\hline \hline Star & $\begin{array}{c}D(\mathrm{Li}) \\
\text { obs }\end{array}$ & $\begin{array}{c}D(\mathrm{Li}) \\
\text { theor }\end{array}$ & $\begin{array}{c}D(\mathrm{Be}) \\
\text { obs }\end{array}$ & $\begin{array}{c}D(\mathrm{Be}) \\
\text { Boesgaard }\end{array}$ & $\begin{array}{c}D(\mathrm{Be}) \\
\text { theor }\end{array}$ \\
\hline HD 4306 & -1.14 & -1.22 & -1.20 & -0.83 & -0.87 \\
HD 26169 & -1.12 & -1.22 & -0.91 & -0.51 & -0.87 \\
HD 27928 & -1.08 & -1.22 & -1.27 & -0.87 & -0.87 \\
HD 45282 & -0.96 & -1.01 & -1.19 & -1.01 & -0.65 \\
HD 108317 & $<-2.03$ & -1.10 & $<-1.72$ & $<-1.38$ & -0.75 \\
HD 126587 & -1.24 & -1.22 & -0.80 & -0.17 & -0.87 \\
HD 128279 & -0.96 & -1.03 & $<-1.09$ & $<-0.67$ & -0.68 \\
HD 200654 & -1.00 & -1.11 & $<-0.95$ & $<-0.46$ & -0.76 \\
HD 218857 & -1.14 & -1.22 & -1.09 & -0.79 & -0.87 \\
HD 274939 & -1.03 & -1.22 & -1.34 & -1.17 & -0.87 \\
BD - 01 2582 & -1.43 & -1.22 & -1.20 & -0.84 & -0.87 \\
CD - 24 ${ }^{\circ} 1782$ & -1.04 & -1.17 & -0.50 & -0.02 & -0.82 \\
CD - 30 0298 & -1.12 & -1.19 & -0.54 & 0.07 & -0.84 \\
\hline
\end{tabular}

First of all, we note that there is now a trend in the logarithmic depletion factor $D(\mathrm{Li})$ (where $D(\mathrm{Li})=A(\mathrm{Li})-A(\mathrm{Li})_{i}$ ) with the effective temperature. This is intrinsically related to the dredge-up mechanism and other observational studies, e.g. Pilachowski et al. (1993), Lèbre et al. (1999) and De Medeiros et al. (2000) have also found a similar depletion pattern. If all the stars have left the main-sequence with the same Li abundance, then our results imply that the cooler subgiants have undergone the larger depletions: cooler means more evolved, hence a more complete dredge-up mechanism, which is in agreement with standard stellar models (e.g. Deliyannis et al. 1990).

Secondly, the observed depletions of our stars seem to agree rather well with the predictions, although the observed 
stars may systematically show somewhat less depletion than the models. We note that $\mathrm{BD}-01^{\circ} 2582$ lies slightly below the general trend, and that HD 108317, denoted by a triangle, seems to be extremely depleted (especially since its abundance is only an upper limit) in lithium compared with the rest of the sample.

Differences between observed and predicted depletion factors seen in Fig. 4 are of the order of 0.1 dex, which may be insignificant, since the Spite plateau value is known with an accuracy hardly better than 0.1 dex. For instance, Ryan et al. (1999) suggested a trend of $A(\mathrm{Li})$ with metallicity, and derived a plateau value of 2.1, whereas Meléndez \& Ramírez (2004) recently found $A(\mathrm{Li})=2.37$, using a newly derived (high) temperature scale. This is just to give an idea of the range of values that one can find in the literature, but it should be kept in mind that these comparisons are only qualitative, as no attempt has been made to put these values on the same scale (i.e. taking into account the fact that different authors may have used different analytical tools, different model atmospheres, different temperature scales).

More relevant to our study which deals with subgiant stars is instead the very recent analysis by Charbonnel \& Primas (2005), whose sample includes not only MS, but also evolved objects. One of their main findings is that the plateau value for their sample of evolved stars is, on average, slightly higher (2.235 or 2.259, depending on the lower $T_{\text {eff }}$ cut-off defining the plateau region, $6000 \mathrm{~K}$ or $5700 \mathrm{~K}$ respectively) than for the dwarfs (2.215 or 2.176 , for the same $T_{\text {eff }}$ cutoffs, respectively). Also, the lithium abundances derived for the evolved stars appear to be slightly more scattered than those for the MS stars. Although these differences are well within the observational errors (as quoted by the authors themselves), if real they would imply a better agreement between observations and predictions for our stars (i.e. differences could be reduced by up to 0.06 dex, e.g. if we had assumed $A(\mathrm{Li})_{i}=2.259$ as our initial lithium abundance).

Clearly, in order to draw firmer conclusions, one should in principle compare the observed depletions to more than just one set of theoretical models. In practice, this is not an easy task, because there have not been simultaneous theoretical predictions for both Li and Be since Deliyannis et al. (1990). For the purpose of this test, Charbonnel (private communication) has kindly provided us with three models computed for a similar stellar metallicity $([\mathrm{Fe} / \mathrm{H}]=-2.27)$ of Deliyannis' model but for three different stellar masses $\left(0.75,0.80\right.$ and $\left.0.85 M_{\odot}\right)$. As one can see from Fig. 5, this new set of models predicts different depletions for different stellar masses. Also, it is interesting to note that depletion sets in at higher temperatures than in the model of Deliyannis: in other words, the same amount of depletion is reached at different temperatures in the two sets of models. This is not completely surprising, as several factors (e.g. initial He content, mixing length, opacities, equation of state) may be slightly different in these models.

In order to make as similar comparison as possible to the dilution curves of Deliyannis et al. (1990), we have interpolated Charbonnel's dilution curves to a stellar mass of $0.775 M_{\odot}$ (dot-dashed curve in Fig. 5). Inspection of this figure shows that higher discrepancies are found between observations and
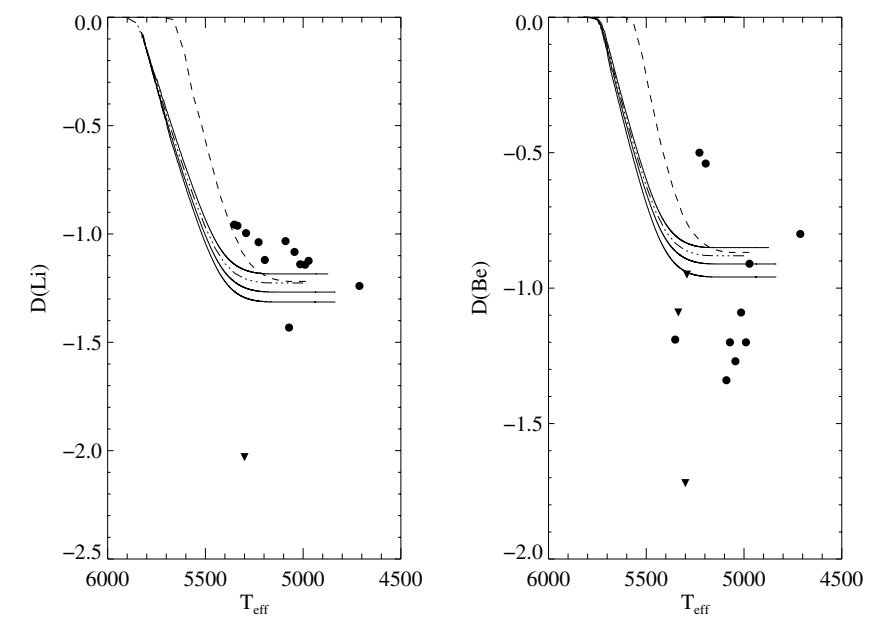

Fig. 5. Observed logarithmic depletion factors (circles) and predictions based on: evolutionary tracks with $[\mathrm{Fe} / \mathrm{H}]=-2.27$ and stellar masses of $0.75,0.80$ and $0.85 M_{\odot}$ (solid lines, from top to bottom) from Charbonnel (priv. comm.) and evolutionary track with $[\mathrm{Fe} / \mathrm{H}]=-2.30$ and stellar mass of $0.775 M_{\odot}$ (dashed line) from Deliyannis. For comparison, an interpolated model from Charbonnel for a stellar mass of $0.775 M_{\odot}$ is also presented (dot-dashed line).

predictions when the interpolated dilution curve is adopted. It seems that for Charbonnel's dilution curves, masses lower than $0.70 M_{\odot}$ would improve the agreement for the majority of our stars.

\subsection{Beryllium depletion}

Our beryllium abundances are plotted versus metallicity in Fig. 6 (top panel), with LTE and NLTE values represented by open and filled circles, respectively. We note that, as in the case of lithium, all our stars have beryllium contents much lower than the general Be evolutionary trend derived from dwarf stars (shown as a dotted line of unitary slope in the figure, cf. Boesgaard et al. 1999). However, since the main process responsible for the formation of beryllium in the Galaxy is cosmic-ray spallation reactions which involve protons and $\alpha$ particles on one side and $\mathrm{C}$ and $\mathrm{O}$ nuclei on the other (Reeves et al. 1970; Meneguzzi et al. 1971), it is more instructive to plot Be versus oxygen instead of iron.

The bottom panel in Fig. 6 shows the correlation between NLTE beryllium and oxygen abundances. The oxygen abundances were derived from the forbidden oxygen line at $630.03 \mathrm{~nm}$ (García Pérez et al. 2005). The best fit we obtained is a linear fit (dashed line, with slope of 0.8). Stars with only upper limits (denoted by triangles) on their Be abundances were excluded from the fit, as well as $\mathrm{CD}-24^{\circ} 1782$ (its oxygen abundance comes from $\mathrm{OH}$ UV lines since its forbidden line was not detected).

As was also the case for lithium, HD 108317 seems to stick out from the rest of the sample, with a beryllium content lower than other stars with similar parameters and oxygen abundances. The observed pattern could imply that this star has undergone a more severe depletion or it may be that its Be abundance before reaching the subgiant evolutionary phase 

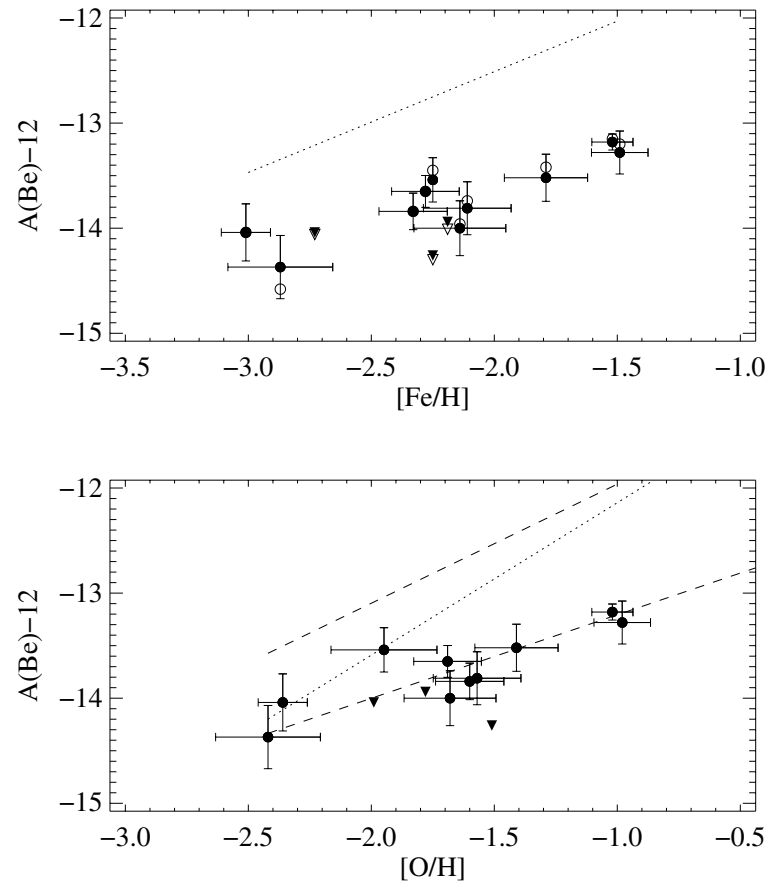

Fig. 6. NLTE and LTE Be abundances as a function of metallicity (top panel) and only NLTE values as a function of oxygen abundances (bottom panel). The dotted lines correspond to: the Be-vs.-Fe (top panel) and the Be-vs.-O (bottom panel) correlation for dwarfs from Boesgaard et al. (1999). The dashed lines correspond to the Bevs.-O correlation for dwarfs from Primas et al. (2005, in preparation, upper line in bottom panel) and for our observed subgiants (lower line in bottom panel).

was lower than for stars of similar oxygen abundances, due to different production scenarios. On the contrary, in the case of $\mathrm{BD}-01^{\circ} 2582$, the beryllium content is comparable to other stars of the sample with similar oxygen abundances, despite its lithium being lower than our derived trend. This is not remarkable, since lithium burns at a lower temperature, i.e. earlier than beryllium if the mixing deepens to hotter stellar layers.

Furthermore, the stars HD 26169 and HD 27928 have very similar $\mathrm{O}$ abundances $([\mathrm{O} / \mathrm{H}] \simeq-1.7)$, but their beryllium contents differ by 0.35 dex. We note that this difference can be only marginally accounted for by the associated observational errors (the final error in our Be abundances is $\simeq 0.20 \mathrm{dex}$ ). As it can be seen from Fig. 7, where the spectra of the two stars are compared, the difference in Be abundances may well be real. Having already measured lithium in both stars, we can now use this important information to check if there is any evidence for different mixing history in these two stars. From Table 2, we see that HD 26169 and HD 27928 have practically identical lithium abundances (within 0.05 dex from each other) and very similar fundamental stellar parameters (cf. Table 1). One could then expect that they have also undergone similar mixing processes: if $\mathrm{Be}$ is affected by a given transport mechanism, then the Li should have changed at least by the same amount. Therefore, the only possible explanation, if the Be abundances are proven to depart significantly in these two stars, is a difference in their initial Be abundances: a production more than a destruction effect.

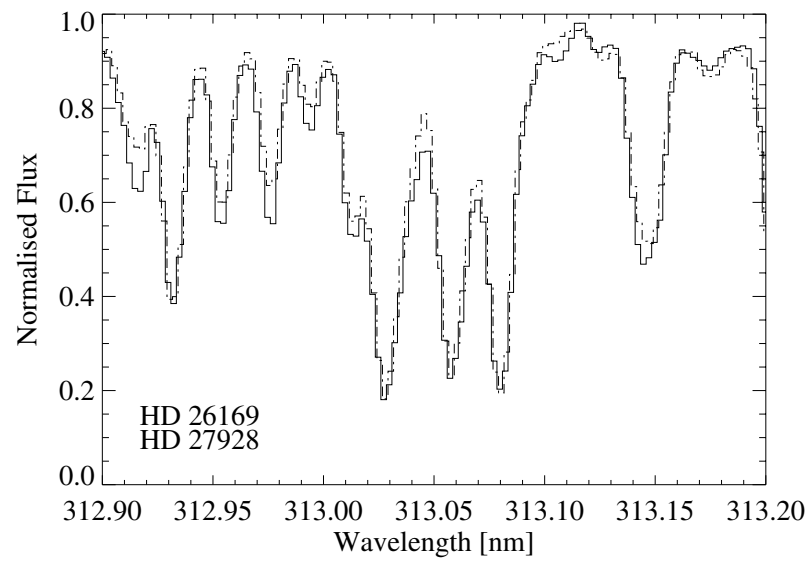

Fig. 7. The UV Be II doublet lines in the observed spectra of HD 26169 (solid line) and HD 27928 (dot-dashed line).

In order to take a look at the global trend of our Be abundances, we now compare the Be-vs.-O correlation we have derived from our subgiant sample with two linear correlations (dotted and upper dashed line, bottom panel, Fig. 6) derived for dwarfs respectively by Boesgaard et al. (1999) and more recently also by our group (Primas et al. 2005, in preparation). We note that the latter analysis finds a non-negligible scatter around the high-metallicity end of the Be-vs.-O relation (which overlaps with our more metal-rich stars) and that both linear and broken fits are under investigation. However, for the purpose of our discussion, and because the data of Primas et al. are still preliminary, we chose to use the simplest of their fits, i.e. the linear one.

Under the assumption that the initial Be abundance of all our subgiants was the one found in dwarf stars, we can now estimate logarithmic Be depletion factors $(D(\mathrm{Be}))$, based on the NLTE Be abundances reported in Table 3. It is important to note that because both dwarf correlations reach only $[\mathrm{O} / \mathrm{H}]=-2.0$ (whereas our sample reaches $[\mathrm{O} / \mathrm{H}] \simeq-2.5$ ), we decided to extrapolate both fits down to our lowest $\mathrm{O}$ abundance in order to be able to compute the $D(\mathrm{Be})$ factors for the entire subgiant sample. The factors thus derived suggest that our stars have depleted Be by approximately 1 dex, as they have evolved off of the main sequence.

Similarly to Li, we compare our estimates (Col. 4, Table 4) with predictions (Col. 6, Table 4) based on depletion calculations kindly provided by $\mathrm{C}$. Deliyannis (private communication). As it is shown in the upper panel of Fig. 8, the standard models predict a Be dilution in subgiants which is expected to depend on effective temperature. However, our depletion factors do not show any obvious sign of such a dependence, although the observational or cosmic scatter may be too large to disclose such a tendency.

Because of the different slopes in the dwarf correlations we have used as a comparison, the emerging trends are quite different. Be seems to be systematically more depleted in our subgiant sample (on average by $0.2 \mathrm{dex}$ ) than expected from the depletion calculations (compare filled with empty circles) if the Primas et al. correlation is used. Instead, when the Boesgaard et al. (1999) relation is considered, the difference between 


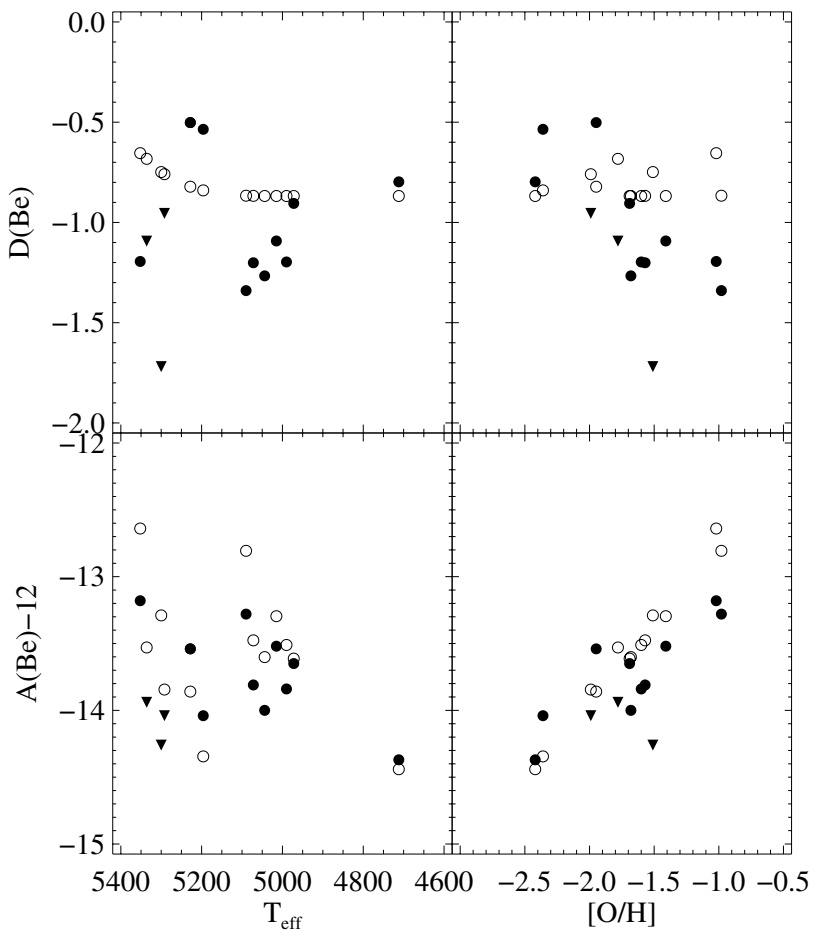

Fig. 8. Logarithmic Be depletion factors (top panels) and $\mathrm{Be}$ abundances (bottom panels) as a function of effective temperature (left panels) and oxygen abundance (right panels). Our estimates (filled circles) are compared with the predictions (open circles). Stars with upper limits on Be abundance are denoted by triangles.

observed and predicted depletion values is reduced (Cols. 5 and 6, Table 4), and for some objects we even observe a reversed behavior, i.e. the models seem to overproduce the amount of depletion that Be has already undergone. Note, however, that the relation of Boesgaard et al. (1999) is based on oxygen abundances derived from $\mathrm{OH}$ UV lines instead of the forbidden oxygen line at $630.03 \mathrm{~nm}$. For these reasons and because we and Primas et al. have used similar methods to derive abundances and stellar parameters, we believe that it is more appropriate to carry out the comparison between MS and subgiant stars in terms of the Primas et al. dwarf relation.

Furthermore, the derived depletion factors do not follow a clean trend but they are quite scattered, especially in a range of $150 \mathrm{~K}$ around $5050 \mathrm{~K}$, where the spread in the Be depletion factors is considerable (with a maximum difference of the order of $0.4 \mathrm{dex}$ ). Although this seems to be at odds with the constant depletion value expected from dilution at these temperatures, the observational errors associated to our Be abundances are quite large. Therefore, the observed scatter cannot be taken as a clear indication of physical differences between the stars.

Possibly, the systematic discrepancies between observed and predicted depletion factors for Be may be the product of our assumption that initial Be abundances are those found in dwarf stars. We explore this possibility by comparing estimates with predictions in a $D(\mathrm{Be})$-vs.- $[\mathrm{O} / \mathrm{H}]$ plot (top-right panel in Fig. 8). We do not see, however, significant differences in depletion discrepancies for different $[\mathrm{O} / \mathrm{H}]$. This suggests that if
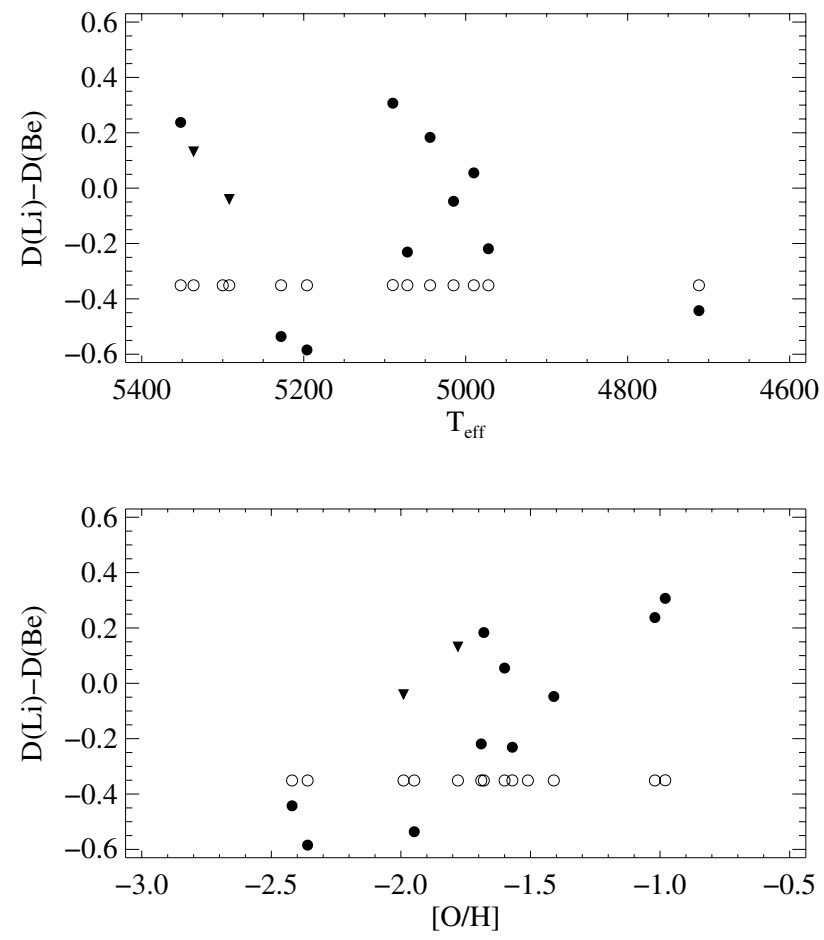

Fig. 9. The differences between logarithmic depletion factors for $\mathrm{Li}$ and $\mathrm{Be}$ as a function of temperature (top panel) and oxygen abundance (bottom panel). Estimates (filled circles) are compared with predictions based on Deliyannis et al. (1990) (open circles).

the assumed values for the initial Be abundance were wrong, the relative errors would be independent of $[\mathrm{O} / \mathrm{H}]$.

According to the tracks of Charbonnel and as it was the case for $\mathrm{Li}, \mathrm{Be}$ depletion depends on the stellar mass. It is also the case here that the Charbonnel's dilution curve interpolated for a stellar mass of $0.775 M_{\odot}$ produces more depletion than the dilution curve of Deliyannis (see right panel in Fig. 5). This clearly helps to reduce the differences between observations and predictions, although this is not enough to eliminate them completely. For most stars in our sample and unlike the case of $\mathrm{Li}$, it would be necessary to resort to dilution curves corresponding to stellar masses higher than $0.775 M_{\odot}$, in order to reconcile our results with the predictions. Furthermore, we note that differences between observations and predictions are much higher than the differences between the dilution curves shown here.

\subsection{Comparison between Li and Be depletions}

Lithium is the most fragile of the three light elements. If the Be content of a star is depleted, it is expected to have already depleted all, or a great fraction of its lithium.

According to standard model predictions (open circles, Fig. 9), Li depletion should be around 0.3-0.4 dex higher than Be depletion. Despite this is a feature common to both sets of dilution curves we have used in our comparisons, most of our observed subgiants (more than 50\%) are characterised by much larger Be depletions (compared to their amount of Li depletion). The stars with a detected Li line but with only upper 
limits for the Be abundance (HD 128279 and HD 200654) are of special interest in this respect.

As already discussed in Sect. 5.1, the agreement between observed and predicted $\mathrm{D}(\mathrm{Li})$ values is at the level of $0.1-0.15$ dex, and it holds for both sets of dilution curves (i.e. Deliyannis and Charbonnel) in the case of the cooler objects of our sample. Thus, it must be beryllium that sticks out: models predict a much smaller amount of depletion, between 0.25 and 0.65 dex, depending on the star under consideration and also on the set of models (for Be, Charbonnel's dilution curves predict a larger amount of depletion for the warmer stars of our sample). Furthermore, one feature that could be worth further investigating is the metallicity dependence of the theoretical dilution curves: in fact, we noted that the best agreement between observed and predicted depletion factors (when both $\mathrm{Li}$ and Be are taken into account simultaneously) is obtained for the two most metal-poor stars of our sample (CD - $24^{\circ} 1782$ and $\mathrm{CD}-30^{\circ} 0298$ ), while the worse agreement is obtained for the most metal-rich stars of our sample (HD 45282 and HD 274939).

Of course, another possibility is that our Be abundances are systematically too low by about 0.2 dex as a consequence of a too low spectrum normalisation (continuum location). This may be directly seen in Fig. 2, where we would not need to change the continuum level very much in order to get a good fit of the observed Be II lines with the lower solid lines. With such Be abundance uncertainty in mind, we should refrain from any definitive statement on departures from the standard depletion calculations, as well as on the possibility that the Be depletion could be larger than that of Li. However, we can make some qualitative speculations.

If our abundances are taken at face value (i.e. without any systematic errors in the Be abundances), we could still reconcile the $\mathrm{Li}$ and $\mathrm{Be}$ depletion data by advocating a higher $\mathrm{Li}$ depletion, e.g. assuming a higher initial lithium of $A(\mathrm{Li})=2.6$. As a matter of fact, the primordial lithium abundance inferred by the baryonic density obtained from WMAP data (Spergel et al. 2003) in combination with standard Big Bang nucleosynthesis (SBBN, Coc et al. 2004) is $A(\mathrm{Li})=2.6$. However, since much lower values are consistently observed in metalpoor dwarf stars (and most of them cluster around $A(\mathrm{Li})=2.2$ ), such a scenario is unlikely. Mixing models like those including rotationally induced mixing (Pinsonneault et al. 2002) indeed suggest some degree of depletion in these stars with respect to the primordial value, the magnitude of which strongly depends on stellar properties such as rotational velocities, masses, temperatures, metallicities etc. However, the absence of any significant scatter in the Li abundances measured in warm halo stars does not favour such a scenario (Bonifacio \& Molaro 1997; Ryan et al. 1999), and it is not obvious whether mixing processes are able to produce such high $\mathrm{Li}$ and Be depletions. We note that theoretical computations including rotation mixing and gravity waves seem to be able to deplete $\mathrm{Li}$ in a very homogeneous way (Talon \& Charbonnel 2004), as required by what is observed in warm halo dwarf stars. Unfortunately, Be depletions have not yet been derived and inspected. The luminosities of our metal-poor subgiants suggest that several of them are starting to ascend the red giant branch so they may already have suffered a first dredge-up. If this process is not well described by standard theory, the predictions of both $\mathrm{Li}$ and Be may be underestimated.

\section{Conclusions}

As stars evolve off the main sequence, their surface convection zone starts to move inwards reaching first $\mathrm{Li}$ depleted and later Be depleted matter. The mixing between these outer and inner layers reduces the surface abundances of these light elements. Subgiants are expected to show signs of such depletion in their $\mathrm{Li}$ and $\mathrm{Be}$ abundances as compared with MS values. Our derived Li abundances lie significantly below the Spite lithium plateau. The depletion is approximately one order of magnitude. A trend with effective temperature may be traced: the warmer stars show the higher Li abundances. In general, these abundances agree well with the standard predictions from evolutionary models of Deliyannis et al. (1990) with the provision that the observations may show somewhat less Li depletion than expected. These differences can be reduced to zero if a value for the Spite plateau 0.1 dex higher were assumed instead, which is quite possible given the uncertainty of its value.

As far as Be is concerned, observations of subgiants show that abundances of $\mathrm{Be}$ and $\mathrm{O}$ are correlated, as in the case of dwarf stars. Be depletion estimates depend on the assumed Be-vs.-O trend of dwarfs, e.g. in our case, that of Boesgaard et al. (1999) and of Primas et al. (2005, in preparation). However, since similar stellar parameters and analytical methods were used here and by Primas et al., we have considered the latter source more appropriate for estimating our Be depletion factors. What emerges is that observed and predicted depletions in our subgiant sample are comparable but in some conflict; the predicted Be depletion is typically $0.3-0.4$ dex smaller than what we observe. We note that a higher initial amount of Li would help reconciling our derived $D(\mathrm{Li})$ and $D(\mathrm{Be})$ depletion factors, so that $D(\mathrm{Li})$ is larger than $D(\mathrm{Be})$ for all our stars. This is challenged by the robustness of the Spite's plateau value of $A(\mathrm{Li}) \simeq 2.2$. A wrong temperature scale could affect this value, but not enough to reconcile our $D(\mathrm{Li})$ and $D(\mathrm{Be})$ values. This would require $A(\mathrm{Li}) \sim 2.6$ which is an unlikely value for the initial Li content of dwarf stars but surprisingly matches the WMAP+SBBN predictions for the primordial Li content. An alternative would be depletion mechanisms acting more efficiently for Be than for $\mathrm{Li}$, mechanisms that are not easy to envisage. However, we note that 0.3 dex could also be a (conservative) estimate of the uncertainty in our Be abundances due to the continuum placement. Hence, should we have systematically performed our spectrum normalisation too low, then a better agreement between standard predictions and observations is obviously obtained.

Similar conclusions can be derived when Charbonnel's dilution curves are used: namely relatively good agreement between observations and predictions of $\mathrm{Li}$ depletion, but somewhat worse in the case of beryllium. According to these dilution curves, our results on Li suggest that the observed subgiants should have masses smaller than $0.75 M_{\odot}$, whereas our observed Be depletion factors suggest that a better agreement could be achieved for stellar masses larger than $0.85 M_{\odot}$. 
Further disentangling between light element observed abundances and theoretical predictions require the availability of a grid of dilution curves, covering the whole range of metallicities of our sample and different masses (since there seem to be a dependence on the stellar mass), as well as higher quality data, in order to reduce the error-bars associated to individual abundance measurements. Should our Be abundances and their depletion factors be confirmed, then this will have important implications on the modelling of the interiors of these stars.

Acknowledgements. The authors are indebted to C. Deliyannis and C. Charbonnel for kindly providing us their $\mathrm{Li}$ and $\mathrm{Be}$ depletion isochrones. A very important part of the work presented here was carried out during the visit of A.E.G.P. to ESO and she is very thankful to ESO for hospitality and financial support. M. Asplund, B. Gustafsson, and C. Charbonnel have contributed significantly to this work with fruitful discussions and valuable suggestions. The work has been supported by the Nordic Optical Telescope and the Swedish Research Council.

\section{References}

Alonso, A., Arribas, S., \& Martinez-Roger, C. 1996, A\&A, 313, 873

Aoki, W., Inoue, S., Kawanomoto, S., et al. 2004, A\&A, 428, 579

Asplund, M., Gustafsson, B., Kiselman, D., \& Eriksson, K. 1997, A\&A, 318, 521

Asplund, M., Grevesse, N., \& Sauval, A. J. 2005, in Cosmic Abundances as Records of Stellar Evolution and Nucleosynthesis, ASP Conf. Ser., 336, 25

Bessell, M. S., Castelli, F., \& Plez, B. 1998, A\&A, 333, 231

Boesgaard, A. M., Deliyannis, C. P., King, J. R., et al. 1999, AJ, 117, 1549

Boesgaard, A. M., \& Tripicco, M. J. 1986, ApJL, 302, 49

Bonifacio, P., \& Molaro, P. 1997, MNRAS, 285, 847

Carlsson, M., Rutten, R. J., Bruls, J. H. M. J., \& Shchukina, N. G. 1994, A\&A, 288, 860

Cassé, M., Lehoucq, R., \& Vangioni-Flam, E. 1995, Nature, 373, 318 Charbonnel, C., \& Primas, F. 2005, A\&A, 442, 961

Coc, A., Vangioni-Flam, E., Descouvemont, P., Adahchour, A., \& Angulo, C. 2004, ApJ, 600, 544

De Medeiros, J. R., do Nascimento, J. D., Sankarankutty, S., Costa, J. M., \& Maia, M. R. G. 2000, A\&A, 363, 239

Dekker, H., D’Odorico, S., Kaufer, A., Delabre, B., \& Kotzlowski, H. 2000, in Optical and IR Telescope Instrumentation and Detectors, ed. M. Iye, \& A. F. Moorwood, Proc. SPIE, 4008, 534

Deliyannis, C. P., Boesgaard, A. M., Stephens, A., et al. 1998, ApJ, 498, L147

Deliyannis, C. P., Demarque, P., \& Kawaler, S. D. 1990, ApJS, 73, 21

Duncan, D. K., Lambert, D. L., \& Lemke, M. 1992, ApJ, 401, 584

Feltzing, S., \& Gustafsson, B. 1994, ApJ, 423, 68
García López, R. J., Severino, G., \& Gomez, M. T. 1995, A\&A, 297, 787

García López, R. J., \& Spruit, H. C. 1991, ApJ, 377, 268

García Pérez, A. E., Asplund, M., Primas, F., Nissen, P. E., \& Gustafsson, B. 2005, A\&A, submitted

Gillis, J. R., Goldman, A., Stark, G., \& Rinsland, C. P. 2001, J. Quantitative Spectroscopy and Radiative Transfer, 68, 225

Gilmore, G., Gustafsson, B., Edvardsson, B., \& Nissen, P. E. 1992, Nature, 357, 379

Grevesse, N., \& Sauval, A. J. 1998, Space Sci. Rev., 85, 161

Hakkila, J., Myers, J. M., Stidham, B. J., \& Hartmann, D. H. 1997, AJ, 114, 2043

Lèbre, A., de Laverny, P., de Medeiros, J. R., Charbonnel, C., \& da Silva, L. 1999, A\&A, 345, 936

Meléndez, J., \& Ramírez, I. 2004, ApJL, 615, 33

Meneguzzi, M., Audouze, J., \& Reeves, H. 1971, A\&A, 15, 337

Michaud, G. 1986, ApJ, 302, 650

Molaro, P., Bonifacio, P., Castelli, F., \& Pasquini, L. 1997, A\&A, 319, 593

Nissen, P. E., Hoeg, E., \& Schuster, W. J. 1997, in ESA SP-402, Hipparcos, Venice '97, 225

Nissen, P. E., Asplund, M., Hill, V., \& D’Odorico, S. 2000, A\&A, 357, L49

Nissen, P. E., Primas, F., Asplund, M., \& Lambert, D. L. 2002, A\&A, 390,235

Olive, K. A., Steigman, G., \& Walker, T. P. 2000, Physics Reports, 333, 389

Parizot, E., \& Drury, L. 1999, A\&A, 349, 673

Pilachowski, C. A., Sneden, C., \& Booth, J. 1993, ApJ, 407, 699

Pinsonneault, M. H., Kawaler, S. D., \& Demarque, P. 1990, ApJS, 74, 501

Pinsonneault, M. H., Steigman, G., Walker, T. P., \& Narayanan, V. K. 2002, ApJ, 574, 398

Primas, F., Duncan, D. K., Pinsonneault, M. H., Deliyannis, C. P., \& Thorburn, J. A. 1997, ApJ, 480, 784

Reeves, H., Fowler, W., \& Hoyle, F. 1970, Nature, 226, 727

Richard, O., Michaud, G., \& Richer, J. 2002, ApJ, 580, 1100

Ryan, S. G., Norris, J. E., \& Beers, T. C. 1999, ApJ, 523, 654

Schramm, D. N., Steigman, G., \& Dearborn, D. S. P. 1990, ApJL, 359, L55

Schuster, W. J., \& Nissen, P. E. 1988, A\&AS, 73, 225

Smith, V. V., Lambert, D. L., \& Nissen, P. E. 1993, ApJ, 408, 262

Spergel, D. N., Verde, L., Peiris, H. V., et al. 2003, ApJS, 148, 175

Spite, F., \& Spite, M. 1982, A\&A, 115, 357

Talon, S., \& Charbonnel, C. 2004, A\&A, 418, 1051

VandenBerg, D. A., Swenson, F. J., Rogers, F. J., Iglesias, C. A., \& Alexander, D. R. 2000, ApJ, 532, 430

Vauclair, S., \& Charbonnel, C. 1995, A\&A, 295, 715

Walker, T. P., Steigman, G., Kang, H., Schramm, D. M., \& Olive, K. A. 1991, ApJ, 376, 51

Walker, T. P., Steigman, G., Schramm, D. N., Olive, K. A., \& Fields, B. 1993, ApJ, 413, 562 